\title{
Trust, Reciprocity, and Guanxi in China: An Experimental Investigation
}

\author{
Fei Song, ${ }^{1}$ C. Bram Cadsby, ${ }^{2}$ and Yunyun $\mathrm{Bi}^{3}$ \\ ${ }^{1}$ Ryerson University, Canada, ${ }^{2}$ University of Guelph, Canada, and ${ }^{3}$ Taiping Asset Management, \\ China
}

\begin{abstract}
We examine the influence of social distance on levels of trust and reciprocity in China. Social distance, reflected in the indigenous concept of guanxi, is of central importance to Chinese culture. In Study 1, some participants participated in two financially salient trust games to measure behaviour, one with an anonymous classmate and the other with an anonymous, demographically identical non-classmate. Other participants, drawn from the same population, completed hypothetical surveys to gauge both hypothetical behaviour and expectations of others. Social distance effects on actual and hypothetical behaviour were statistically consistent. The results together corroborated the hypothesized negative relationship between trust and social distance. However, reciprocity was not responsive to social distance. Study 2 found that affect-based trust, but not cognition-based trust, played a mediating role in the relationship between social distance and interpersonal trust in a hypothetical scenario. We conclude that close guanxi ties in China engender affect-based trust, which is extended to shouren classmates. This is true despite the fact that no more cognition-based trust is placed nor reciprocity received or expected from classmates compared to demographically identical shengren non-classmates.
\end{abstract}

KEYwORDS affect-based trust, China, guanxi, reciprocity, trust

\section{INTRODUCTION}

Trust and reciprocity are the glue of social exchange (Granovetter, 1985; Pruitt \& Kimmel, 1977; Yamagishi, 1998). As many social and economic transactions take place sequentially, one party must transfer valuable resources to another before obtaining anything in return and the other party must honour an obligation that might be in its short-term interest to ignore. In theory, such obligations can be enforced by formal contracts and sanctioning systems. In practice, however, imperfect monitoring, information asymmetries, moral hazard, and transaction costs often make the use of binding contracts infeasible or prohibitively costly. As a result, trust and reciprocity are essential in human affairs: without trust and 
reciprocity, many beneficial social exchanges would not begin and without reciprocity they would not end, to the detriment of the involved parties in particular and society in general.

This article examines the role of social distance (Akerlof, 1997) and the relational closeness of the interacting parties in determining trust and reciprocity. We examine this in the context of China for several reasons. First, cross-cultural research holds China to be the paragon of highly collectivist societies (e.g., Hofstede, 1991), in which boundaries of trust are limited to narrow ingroups (Bond, 1991; Triandis, 1995). Though trust everywhere involves cognitive and affective bases, comparative studies find that in China the relationally relevant affective bases are more salient (Wasti, Tan, \& Erdil, 2011) and that the two types of trust are more intertwined (Chua, Morris, \& Ingram, 2009). Moreover, it has been suggested that the obligations of reciprocity in collectivist societies permeate many facets of life and are difficult to break (Wasti et al., 2011). In sum, the comparatively greater salience of relational concerns in China makes it a good setting in which to identify influences of relational closeness on trust and reciprocity.

Second, the relevance of social distance is recognized in indigenous Chinese folk psychology. The ubiquitous term guanxi literally means relationships, and refers to particularistic ties rooted in a common background and experience that facilitate exchange (Tsui \& Farh, 1997). Management researchers have explored different ways to operationalize the concept of guanxi and examine its impact on various interpersonal interactions in China (e.g., Chai \& Rhee, 2010; Farh, Tsui, Xin, \& Cheng, 1998; Luo, 2000; Tsui \& Farh, 1997; Xin \& Pearce, 1996; Yang, 1994). We join this effort by offering another approach, examining how trust and reciprocity in economic games varies as a function of guanxi-relevant relationship categories.

Another goal of this research is to investigate the convergent validity of two common ways of measuring trust and reciprocity - behavioural games with real economic incentives versus hypothetical scenarios about such games. The use of behavioural measures with salient financial incentives in this area of research has been strongly endorsed by some behavioural psychologists (e.g., Hogarth \& Reder, 1987), behavioural/experimental economists (Camerer, 2003; Rabin, 2002; Smith, 1976), and social-exchange sociologists (Yamagishi, 1998). However, hypothetical measures have also been widely used by some economists (e.g., Buchan \& Croson, 2004) and many managerial and social psychologists (e.g., Mayer, Davis, \& Schoorman, 1995; Robinson, 1996; Rotter, 1967). It is unclear at present whether these two types of measures tap the same underlying psychological processes. Earlier research shows that being psychologically removed from a situation can make it difficult to imagine how behaviour might be influenced by emotional or visceral forces that are felt in an actual situation (Loewenstein, 1996; Van Boven, Dunning, \& Loewenstein, 2000). In such circumstances, it may be difficult for individuals to predict correctly how they would act. Two recent studies discussed below, one a laboratory experiment with financial incentives (Buchan, Johnson, \& Croson, 
2006), and the other hypothetical (Buchan \& Croson, 2004), report contradictory results on the effect of social distance on trust and reciprocity in China. However, these studies differ along other dimensions as well. Thus, it is not clear whether their different conclusions arise from a difference between behavioural and hypothetical measures or some other difference in procedures. To investigate this, the present research studies the effect of social distance under both conditions - behavioural games with real incentives versus hypothetical scenarios about such games randomly assigning participants to these conditions so as to examine the effects.

\section{TRUST, RECIPROGITY, AND GUANXI}

We build on the widely used definition of trust by Rousseau, Sitkin, Burt, and Camerer (1998: 395): 'a psychological state comprising the intention to accept vulnerability based upon positive expectations of the intentions or behaviour of another'. In our study, this definition is broadened to include both the expression of a hypothetical intention to accept such vulnerability and explicit behaviour resulting from such an intention. Correspondingly, we adopt a definition of reciprocity as the act of "voluntarily repaying a trusting move at a later point in time, although defaulting on such repayment is in the short-term self-interest of the reciprocator' (Gunnthorsdottir, McCabe, \& Smith, 2002: 50). In trust and reciprocity exchanges, one party's action prompts another's response, the anticipation of which in turn affects the first party's action. Hence, a game-theoretic approach, analyzing strategic decision situations with an explicit emphasis on exchange, conflict, and interdependence, is appropriate for studying such a phenomenon (Murnighan, 1994). To this end, we use a variant of the widely employed 'trust' game (Berg, Dickhaut, \& McCabe, 1995; see Camerer, 2003; Wilson \& Eckel, 2011; Garbarino \& Slonim, 2009, for extensive reviews of the large empirical literature using this game) to model and measure trust and reciprocity. Participants are randomly assigned to be either trustors or trustees and given identical monetary endowments at the beginning of the game. Each trustor then decides how much of his/her endowment to send to an anonymous counterpart (trustee), with whom $\mathrm{s} /$ he is paired at random. Any amount from zero to the entire endowment may be sent. All participants are informed that the experimenter will triple the amount sent before it is passed on to the trustee. This tripling represents the benefits of trust to those participating in a trust/reciprocity exchange. After receiving the money, each trustee then decides how to split his/her total wealth, i.e., the sum of his/her initial endowment plus the tripled amount received, between him/herself and the trustor as an act of reciprocity. Each trustee is permitted to divide his/her posttransfer level of wealth in any manner s/he desires between him/herself and the trustor with whom s/he was randomly matched.

Non-cooperative game theory predicts that trustees, if self-interested, rational, and motivated to maximize their wealth, would send no money back to trustors. 
Since there is no way of penalizing such self-interested behaviour, rational trustors should then accurately expect trustees to send nothing back. Therefore, trustors have no motivation to send anything to trustees in the first place. Thus the subgame perfect Nash equilibrium predicts neither trust nor reciprocity behaviour in such a setting. In stark contrast, both trust and reciprocity are observed in behavioural studies where people play these games; the vast majority of trustors send non-trivial amounts to trustees, who in turn send non-trivial amounts back. Average amounts sent have been documented to range from 40 to 60 percent of trustor endowments, while amounts returned average 110 percent of the amount originally sent (Camerer, 2003). Such patterns have been attributed to aspects of human psychology beyond narrow self-interest such as moral sentiments and the motivation to uphold social norms. However, investigations of factors moderating trust and reciprocity are needed to test such explanations more incisively.

Guanxi is an idea familiar to virtually every Chinese person. It expresses the central importance of social relationships within Chinese society. Of course, relationships are important in economic interactions in both Western and Chinese societies (e.g., Burt, 1992; Luo, 2000; Tsui \& Farh, 1997; Xin \& Pearce, 1996). Yet many scholars contend that relationships are more important to trust in China, in part because there may be less generalized trust - trust extended to all other people (e.g., Bian, 1997; Chen \& Chen, 2004; Su \& Littlefield, 2001). For Chinese people it is argued that the level of trust depends on the category of relationship one has with another person (e.g., Butterfield, 1982; Chen \& Chen, 2004). Put differently, trust toward members of one's ingroup and distrust toward others is one of the strongest characteristics of interpersonal relations in China (Yang, 1994). Thus, trust is considered very important in China and yet at the same time it is difficult to build trust beyond fairly restrictive circles (e.g., Fukuyama, 1995).

How do people build guanxi? Chiao (1982) and King (1991) suggest that Chinese guanxi is often based on shared social experiences and associations, such as with relatives or classmates. According to Yang (1994), guanxi also implies 'social connections' built implicitly, without the need for explicit discussion or arrangements, upon mutual interest and benefits. Once two people have established a sufficient level of guanxi, each can request a favour from the other knowing that the opportunity to reciprocate will arise at an appropriate time in the future. Tsui and Farh (1997) further note that such interpersonal favours and acts of generosity are rendered with the anticipation that they will be reciprocated. Moreover, Xin and Pearce (1996) found executives use guanxi connections to reduce threats to their business such as extortion or appropriation. Other recent empirical works also show that guanxi is critical for achieving entrepreneurial success in China (c.g., Guo \& Giacobbe-Miller, 2010; Li, Yao, Sue-Chan, \& Xi, 2011). Theorists propose that guanxi functions as a substitute for legal protections, providing 'contextual confidence' for building trust (Child \& Mollering, 2003). 
Given the time-scale over which guanxi operates, it is important to test its effects as a function of real relationships rather than relationships contrived in the laboratory. Our research was conducted with undergraduate business students at the Dongbei University of Finance and Economics (DUFE) and the Shanghai University of Finance and Economics (SHUFE). At DUFE and SHUFE, as at many other Chinese universities, four classmates generally share a dorm room during the four years spent at a university. Females from the same class are all in adjacent rooms, as are males. They take almost all of their classes together. Jacobs (1979) found that being classmates is a base for guanxi. Su and Littlefield (2001) place classmates at the more specific level of shouren guanxi, a close category that also includes relatives other than immediate family, friends, teachers, neighbours, and co-workers. Although two students from the same university who are from different classes and do not know each other might share some guanxi by virtue of attending the same university, the level of such guanxi would be considerably lower than between two classmates (Jacobs, 1979). In particular, their relationship would be comparable to employees in the same large company, whom Su and Littlefield (2001) place in the more distant category of shengren guanxi.

\section{Hypotheses}

Since classmates are shouren and university-mates merely shengren, classmates should garner more trust. Moreover, Farh et al. (1998) show that among 32 business executives in China, being former classmates was one of three guanxi variables that was significantly related to the expression of more interpersonal trust. Thus, we expect more trust among classmates than among non-classmates. Since classmates and non-classmates are in the same demographic category, relational demography theory provides no reason to trust one more than the other. Moreover, although personal feelings between two specific individuals who know each other can certainly have a strong impact on guanxi as well as on trust and reciprocity behaviour, no such feelings can play a role in our anonymous study. Indeed there is no reason to expect any difference in character or personality between a classmate and a non-classmate. Thus, our first hypothesis focuses solely on the role played by the guanxi category in affecting the level of trust.

Hypothesis 1a: Trustors will exhibit a higher level of trust toward classmates than toward non-classmates.

Hypothesis 1b: Trustees will expect a higher level of trust from classmates than from non-classmates.

The arguments underlying our next two hypotheses arise out of similar considerations to those underlying the first. Since classmates are shouren, while non- 
classmates are shengren, one might expect a more generous reciprocity response from a classmate than from a non-classmate. Moreover, if reciprocity responses were more generous from classmates than from non-classmates, it would provide a rationale for placing more trust in classmates as is predicted by Hypothesis 1. Hence the second hypothesis proposes an impact of social distance on reciprocity, measured by money returned over money sent (e.g., Berg et al., 1995; Camerer, 2003; Song, 2008, 2009).

Hypothesis 2a: Reciprocity (the ratio of money returned to that sent) will be higher from classmates than from non-classmates.

Hypothesis 2b: Trustors will expect higher reciprocity from classmates than from nonclassmates.

Contrasting with the second hypothesis, a different strand of theory leads to an alternative conjecture. The reciprocity decision is quite different from the initial decision to trust. Trusting involves exposure to an uncertain outcome; a trustor must always be aware of the possibility of betrayal once trust is extended. A trustee faces no such uncertainty and no such financial risk. S/he has received a sum of money from an anonymous trustor and must simply decide how much money to return to the trustor as a reciprocating gesture. Since there is no uncertainty about the outcome, there is little reason to concern oneself with the likely reaction of the anonymous trustor. Thus, the reciprocity decision might be less influenced by social distance or guanxi. This logic is similar to Tsui and Farh's (1997: 62) proposal that guanxi 'may play a more significant role in Chinese organizational behaviour when task uncertainty is high than when it is low'. Moreover, Su and Littlefield (2001: 203) argue that if a shengren takes 'the lead to make the commitment to a common interest', this behaviour will be appreciated and 'cannot be explicitly refused' according to the principle of renqing, which involves the exchange of favours and generosity. Hwang (1987) emphasizes that the principle of renqing places an obligation on the recipient of a gift to reciprocate to the donor. Thus, whether a gift is received from a classmate shouren or from a non-classmate shengren, the same reciprocation would be expected. Hence the contrasting hypotheses:

Hypothesis 3a: Reciprocity will not differ between classmates and non-classmates.

Hypothesis 3b: Trustors' expectations of reciprocity will not differ between classmates and non-classmates.

We have considered trust in terms of sending money in trust games, another approach considers trust as social perceptions/intentions toward another. 
Organizational researchers have distinguished between two types of trust in relationships: cognition- and affect-based trust (e.g., Lewicki \& Bunker, 1995; McAllister, 1995; Ng \& Chua, 2006). Cognition-based trust involves perceptions that another person has the competency and integrity to be trustworthy, while affect-based trust is based on the emotional bond and concerns one feels toward the other person - simply put, trust from the head versus trust from the heart (Chua et al., 2009). Empirical research has provided evidence of discriminant validity for these two types of trust ( $\mathrm{Ng} \&$ Chua, 2006) and has shown that they can lead to different behaviours and reactions (Levin \& Cross, 2004; Ng \& Chua, 2006). Chua et al. (2009) argued that in China affective bonds among business partners are especially important relative to comparable relationships in the United States and further demonstrated that cognition-based trust is more intertwined with affect-based trust among Chinese than among American managers. This particular conceptualization of trust is closely related to the discussion of guanxi by Chen and Chen (2004), who argue that guanxi quality is predicted by both trust (xin) and feeling (qing), where trust is primarily cognition-based and feeling affect-based. Thus, for Chinese people guanxi quality derives from a combination of both cognition-based and affect-based factors, which are closely related to the two types of trust discussed above. The important role of affect in Chinese organizations is evident in many organizational practices. For example, many companies in China hold regular festival parties and organize vacation trips for employees to socialize with each other. Co-workers and colleagues often know each other's family and friends well, cultivate friendships, and socialize with each other after work. Thus, we might expect that trusting behaviour related to guanxi in China will have an affective basis.

Our studies test this in a circumstance where this affective basis cannot be confounded with a cognitive basis as it might in many real world contexts. We compare trust between classmate shouren and non-classmate shengren at the same university. Since the classmates and non-classmates in our study are demographically alike (i.e., same university, similar education, similar age), there would be little variance in factors relevant to cognition-based trust. Given the anonymous nature of the interaction, participants cannot apply knowledge of the individual personality and behavioural history of their counterpart. Also, the anonymous nature of the game eliminates the factor of anticipated social sanctioning, isolating the role of positive affective feelings that come with a close shengren relationship. Thus, we propose the following hypotheses:

Hypothesis 4a: Affect-based trust will be greater for classmates than for non-classmates, but there will be no such difference in cognition-based trust.

Hypothesis 4b: Affect-based trust will mediate the effect of classmate/non-classmate on trust. 


\section{METHOD - STUDY 1}

Using the experimental framework of the trust game, this study examines the effects of three manipulated factors. The first is a between-participants factor: the random assignment of participants to the behavioural games with salient financial incentives or hypothetical survey scenarios about games, permitting the gathering of these two types of data separately with no cross-contamination, and allowing comparisons across these response formats in participants' decisions and their expectations about counterparts. (Note that comparing such response formats in a within-participants design would render any such comparison suspect since most people would likely report hypothetical beliefs consistent with their actual behaviour.)

The second and most important factor is a within-person manipulation of social distance, involving interaction with a classmate in one's own curricular track vs. a non-classmate who attends the same university but takes different classes on different subjects. This within-person factor is used to control for individual differences in general trust/reciprocity preferences when examining the impact of social distance on trust and reciprocity. The third manipulation is a between-person factor: the order of the within-person social-distance manipulation. The order is reversed and this factor is counterbalanced to isolate the social-distance effect from potential order effects.

The 234 participants majored primarily in Business English or Public Administration at DUFE and all had taken similar courses related to economics, so their levels of exposure to game theory were equivalent. The behavioural session consisted of 116 ( 79 women and $37 \mathrm{men}$ ) participants with an average age of 20.79 $(\mathrm{SD}=0.95)$. The hypothetical session consisted of 118 participants ( 80 women and $38 \mathrm{men}$ ) with an average age of 20.41 ( $\mathrm{SD}=0.93$ ). Everyone directly involved in conducting the experiment was Chinese to avoid any effect of foreign involvement on behaviour.

Behavioural session procedure. Participants from two different university classes arrived at the experiment site. Each participant was asked to pick an identification card out of a box, which determined his/her participant code and assignment to either the 'Party A' ('Fiafang' in Chinese) or 'Party B' ('Yifang' in Chinese) role, corresponding to trustor and trustee, respectively. In order to avoid possible framing effects, the word 'trust' was not mentioned at all during the experiment. Participants were then escorted to the assigned 'Party A' or 'Party B' room for their class, where they stayed for the remainder of the session. Thus, participants sat in one of four rooms assigned on the basis of both class and role in the experiment and did not meet each other during the experiment. All participants received the same general instructions about the trust game. They were informed that the experiment involved the game described above in which they would 
either play the 'Party A' or 'Party B' role. The game was illustrated with several numerical examples in the instructions. The instructions were read aloud to the participants and they were then given time to ask questions. Participants were also told that they would remain anonymous during the experiment (they were only identified by their unique participant codes), and that they would get paid in cash at the end of the game based on the decisions they made and those made by another participant with whom they would be randomly paired during the game. Participants were asked to complete a quiz containing a numerical example to ensure that they completely understood the game.

The experiment began at that point. Each trustor and trustee received a $¥ 20.00 \mathrm{RMB}$ endowment at the beginning of each of the two experimental rounds. A decision record form was employed for trustors and trustees to communicate their decisions anonymously to each other. The decision record forms were delivered in envelopes. One experimenter or research assistant was permanently stationed in each room, while two additional assistants collected and delivered the decision record forms between the rooms. Finally, another research assistant sat in the control room recording all the decisions by participant code in isolation from the participants themselves. Thus, no one could link a participant code with a name or observe the decisions made. This decision communication procedure minimized potential confounding effects emanating from self-presentation and/or social desirability motivations.

Participants made two decisions sequentially, interacting with different people. Half of the participants interacted first with a classmate and then with a nonclassmate counterpart; while for the other half this order was reversed (CN and NC order hereafter). In addition to this reversal, the following procedures were implemented to mitigate multiple-round effects. First, participants were not told the number of decisions they would be asked to make at the beginning of the experiment, nor were they informed in the second condition that it was the last condition in the experiment. Second, the outcomes for the first condition were not revealed to the trustors until the very end of the experiment, i.e., after they completed the second condition and the post-experiment questionnaire. However, due to the game structure, a trustee always knew the result of an interaction as soon as $s /$ he made a decision.

At the end of the experiment participants were asked to complete a short post-experiment questionnaire for information on gender and age. After completing the questionnaire, participants were paid individually in the experimental control room to protect their anonymity. The research assistants who paid the students had not worked on any other aspect of the experiment and were unfamiliar with the structure of the game. Thus, they were unable to infer anything about the decisions made by the participants from the amounts of money earned. Only one of the two experimental rounds was randomly chosen for payment to ensure that money earned in one round did not affect behaviour in the subsequent 
round and that each round was considered independent and equally important. Each session took approximately one hour and participants earned on average $¥ 27.50 \mathrm{RMB}$. This was substantially higher than the average wage of $¥ 10 \mathrm{RMB}$ to $¥ 15 \mathrm{RMB}$ an hour for jobs on campus and had purchasing power equivalent to about US\$18.

Hypothetical session procedure. The hypothetical questionnaire session followed the same procedures employed in the behavioural session as much as possible. Participants were required to complete two separate questionnaire surveys one by one. In each questionnaire, after following along as the experimenter read aloud the instructions for the behavioural trust game summarized above, participants were asked to respond to the following questions translated here from the Chinese:

Trustors: Imagine you were playing this game as 'Party A'. Please state how much you would send to a randomly-paired 'Party $B$ ', who is your fellow classmate (or a non-classmate) from $D U F E$, and how much you would expect to receive back from $\mathrm{him} / \mathrm{her}$ based on the amount you would send.

Trustees: Imagine you were playing this game as 'Party B'. Please state how much you would expect to receive from a randomly-paired 'Party $A$ ', who is your fellow classmate (or a non-classmate) from DUFE, and how much you would return to him/her based on your expectation.

At the end of the experiment participants were asked to complete the same post-experiment questionnaire as in the behavioural session. Since the hypothetical sessions involved purely hypothetical endowments, participants were paid a fixed participation fee of $¥ 10 \mathrm{RMB}$ for about 45 minutes of their time.

\section{RESULTS - STUDY 1}

Table 1 summarizes the results of both the behavioural and hypothetical format conditions. Specifically, we report the means and standard deviations for behavioural, hypothetical, and expected trust and reciprocity toward both a classmate and a non-classmate. In addition, we report one-sample $t$-tests of the within-person difference in trust and reciprocity decisions with classmate and non-classmate counterparts. The $P$-values associated with these $t$-tests indicate that trust (the amount of money sent to the trustee) were all significantly higher toward a classmate than toward a non-classmate for behavioural, hypothetical, and expectation measures $(p=0.02,0.00$, and 0.00 respectively), while there was no corresponding differences in reciprocity decisions (the amount of money sent back to the trustor). 
Table 1. Means and standard deviations by experimental treatment (Study l)

\begin{tabular}{|c|c|c|c|}
\hline Variable & Classmate $^{\dagger}$ & Non-classmate ${ }^{\dagger}$ & $\begin{array}{l}\text { One-sample } t \text {-test of the } \\
\text { within-person difference } \\
\text { (p-value in parentheses) }\end{array}$ \\
\hline \multicolumn{4}{|l|}{ Panel A: Behavioural Format } \\
\hline Trust Decision $(\mathrm{n}=58)$ & $9.65(6.35)$ & $7.34(6.32)$ & $2.50(0.02)$ \\
\hline Reciprocity Decision $(\mathbf{n}=50)$ & $1.65(1.09)$ & $1.45(1.03)$ & $0.45(0.66)$ \\
\hline \multicolumn{4}{|c|}{ Panel B-1: Hypothetical Format, Trustors } \\
\hline Trust Decision $(n=59)$ & $11.36(6.44)$ & $8.05(5.51)$ & $5.87(0.00)$ \\
\hline Reciprocity Expectation $(n=59)$ & $1.77(0.52)$ & $1.64(0.96)$ & $0.12(0.22)$ \\
\hline \multicolumn{4}{|c|}{ Panel B-2: Hypothetical Format, Trustees } \\
\hline Trust Expcctation $(\mathrm{n}=59)$ & $9.32(6.39)$ & $5.38(4.33)$ & $5.18(0.00)$ \\
\hline Reciprocity Decision $(\mathrm{n}=59$ ) & $1.88(0.55)$ & $1.68(0.65)$ & $0.33(0.13)$ \\
\hline
\end{tabular}

Notes:

'Standard deviations are in parentheses.

To formally test Hypotheses 1 to 3, we conducted a 2 (within-person repeated measure of social distance level) $\times 2$ (between-person measure of decision-order) mixed analysis of variance (mixed ANOVA) test for each dependent variable. ${ }^{[1]}$ Table 2 summarizes the results of this analysis.

For trust decisions measured behaviourally, a significant main effect was found for level of social distance $[\mathrm{F}(1,56)=6.21, \mathrm{p}=0.02]$. This implies that participants, averaged over the CN and $\mathrm{NC}$ orders, exhibited significantly higher trust toward a classmate than toward a non-classmate. Order had no significant interaction with the main effect of social distance on trust. For the hypothetical measure, trust decisions showed a significant main effect of social distance on both hypothetical trust $[\mathrm{F}(1,57)=34.05, \mathrm{p}=0.00]$ and expected trust $[\mathrm{F}(1,57)=28.90, \mathrm{p}=0.00]$. The measure of expected trust also showed an interaction effect between social distance and order, indicating that social distance had a slightly stronger effect in the $\mathrm{NC}$ than in the $\mathrm{CN}$ order $[\mathrm{F}(1,57)=4.61, \mathrm{p}=0.04]$. A corresponding dummyvariable regression analysis, not reported in detail to save space, confirmed that social distance was nonetheless significantly related to expected trust regardless of order ( $p=0.03$ for $\mathrm{CN}$ order, $\mathrm{p}=0.00$ for $\mathrm{NG}$ order). These results together provide strong support for Hypotheses $1 \mathrm{a}$ and $\mathrm{lb}$.

In contrast to these findings about trust, tests of reciprocity measures did not show effects of social distance. For reciprocity decisions measured behaviourally, there were no main effects or interactions between order and level of social distance. We controlled for the level of trust received, ${ }^{[2]}$ and this factor did not interact with social distance to determine reciprocity either. For hypothetical measures, we conducted parallel analyses and found that neither reciprocity choices nor expectations showed effects of social distance, nor its interactions with order or with trust received. Indeed, there was no correlation between the 
Table 2. Mixed ANOVA testing effects of social distance and order (Study l)

\begin{tabular}{|c|c|c|c|c|}
\hline Variable and Source & $d f$ & $F$ & p-value & Partial $\eta 2$ \\
\hline \multicolumn{5}{|c|}{ Panel A: Trust Decision - Behavioural Format } \\
\hline Social Distance & 1 & 6.21 & 0.02 & 0.10 \\
\hline Social Distance*Order & 1 & 0.07 & 0.79 & 0.00 \\
\hline Error & 56 & & & \\
\hline \multicolumn{5}{|c|}{ Panel B: Reciprocity Decision - Behavioural Format } \\
\hline Social Distance & 1 & 0.17 & 0.69 & 0.00 \\
\hline Social Distance*Trust Received & 1 & 0.01 & 0.92 & 0.00 \\
\hline Social Distance*Order & 1 & 1.72 & 0.20 & 0.04 \\
\hline Error & 47 & & & \\
\hline \multicolumn{5}{|c|}{ Panel C: Trust Decision -Hypothetical Format } \\
\hline Social Distance & 1 & 34.05 & 0.00 & 0.37 \\
\hline Social Distance*Order & 1 & 0.52 & 0.47 & 0.01 \\
\hline Error & 57 & & & \\
\hline \multicolumn{5}{|c|}{ Panel D: Reciprocity Decision-Hypothetical Format } \\
\hline Social Distance & 1 & 0.05 & 0.82 & 0.00 \\
\hline Social Distance*Trust Expected & 1 & 1.44 & 0.24 & 0.03 \\
\hline Social Distance*Order & 1 & 2.70 & 0.11 & 0.05 \\
\hline Error & 56 & & & \\
\hline \multicolumn{5}{|l|}{ Panel E: Trust Expectation } \\
\hline Social Distance & 1 & 28.90 & 0.00 & 0.34 \\
\hline Social Distance*Order & 1 & 4.61 & 0.04 & 0.08 \\
\hline Error & 57 & & & \\
\hline \multicolumn{5}{|l|}{ Panel F: Reciprocity Expectation } \\
\hline Social Distance & 1 & 1.48 & 0.23 & 0.03 \\
\hline Social Distance*Trust Received & 1 & 2.24 & 0.14 & 0.04 \\
\hline Social Distance*Order & 1 & 2.32 & 0.13 & 0.04 \\
\hline Error & 52 & & & \\
\hline
\end{tabular}

reduction in trust received and the unchanging reciprocity ratio when moving from the classmate to the non-classmate level. Moreover, there was no significant correlation between behavioural trust and actual reciprocity or hypothetical trust and expected reciprocity between persons at either the classmate or non-classmate level either.

As a robustness check of the reciprocity findings, we used the absolute amount sent back rather than the ratio of amount sent back over amount sent, while controlling for the amount sent, as an alternative measure of reciprocity. This enabled us to include those instances in which no money was sent by the trustors. The results remained qualitatively identical to those using the reciprocity ratio: Even when we drop the control for increased trust received at the classmate versus the non-classmate level, social distance has no significant effect on the level of reciprocity. Overall, results from the behavioural and hypothetical conditions do not confirm Hypothesis 2 and are consistent with Hypothesis 3. 


\section{METHOD - STUDY 2}

Our second study was motivated by a puzzle in the findings of Study 1 . While more trust was extended to classmates than to non-classmates, non-classmates were just as trustworthy as classmates as measured by their reciprocity behaviour. Indeed, trustors did not even expect a significantly greater return on their trust investment from classmates than from non-classmates. This raises the question of why trustors exhibit more trust in classmates than in non-classmates when they neither expect nor receive better reciprocity treatment from the former than from the latter. Study 2 investigated our hypothesized answer to this question, which focuses on the distinction between affect- and cognition-based trust. ${ }^{[3]}$ Since there were no significant differences between the results of our behavioural and hypothetical treatments in Study 1, we employed only the hypothetical methodology in Study 2.

Sample and procedures. Sixty undergraduate business-major students at SHUFE participated in the study. The participants were in the third year of their undergraduate study and 40 percent were male. The study consisted of two parts. Part one was completed a week before part two. In part one, participants completed two questionnaires in which they were asked to consider all the questionnaire items in relation to one of their classmates on one questionnaire and to someone from the same university but a different class on the other. Half of the participants received the questionnaires in one order; half received them in the other order. The questionnaires stated that 'you do not know specifically who this person is, but you do know that it is one of your classmates/ someone from the same university but not the same class'. Affect- and cognition-based trust were each assessed using six items adopted from McAllister (1995). Sample items include: 'We can both freely share our ideas, feelings, and hopes'; and 'We would both feel a sense of loss if one of us was transferred and we could no longer work together' for affect-based trust, and 'I can rely on this person not to make my job more difficult by careless work' and 'This person approaches his/her job with professionalism and dedication' for cognition-based trust. We also measured the shouren/shengren construct with six items adopted from Webber, Chen, Marsh, and Payne (1999). Specifically, we asked participants to rate their familiarity with the academic reputation, strengths, and weaknesses of a typical classmate and non-classmate (from the same University). Sample items include 'I know the academic reputation of this person well' and 'I am familiar with the way s/he works'. Lastly, we measured the interaction frequency as another measure of shouren/shengren guanxi (Webber et al., 1999). Sample items include 'I initiate interaction with him/her frequently' and 'S/he initiates interaction with me frequently'. We used a seven-point Likert scale for all items with responses ranging from 1 (strongly disagree) to 7 (strongly agree). All items were in Chinese and had gone through a translation and back-translation 
Table 3. Means and standard deviations by experimental treatment (Study 2)

\begin{tabular}{|c|c|c|c|}
\hline Variable & Classmate $^{\dagger}$ & Non-classmate ${ }^{\dagger}$ & $\begin{array}{l}\text { One-sample t-test of the } \\
\text { within-person difference } \\
\text { (p-value in parentheses) }\end{array}$ \\
\hline \multicolumn{4}{|l|}{ Data from the Trustors } \\
\hline Affect-Based Trust & $4.92(1.30)$ & $3.97(1.44)$ & $3.19(0.00)$ \\
\hline Cognition-Based Trust & $4.63(1.15)$ & $4.34(1.14)$ & $1.23(0.23)$ \\
\hline Shengren-shouren Guanxi & $4.82(1.35)$ & $3.19(1.29)$ & $6.42(0.00)$ \\
\hline Interaction Frequency & $5.50(1.16)$ & $4.00(1.35)$ & $2.67(0.01)$ \\
\hline Trust Decision & $12.42(7.86)$ & $6.70(4.93)$ & $4.72(0.00)$ \\
\hline Reciprocity Expectation & $1.51(0.43)$ & $1.41(0.49)$ & $0.81(0.42)$ \\
\hline \multicolumn{4}{|l|}{ Data from the Trustees } \\
\hline Affect-Based Trust & $4.43(1.11)$ & $3.53(1.45)$ & $3.34(0.00)$ \\
\hline Cognition-Based Trust & $4.62(0.96)$ & $4.49(1.01)$ & $1.15(0.26)$ \\
\hline Shengren-shouren Guanxi & $4.61(1.09)$ & $3.18(1.38)$ & $5.88(0.00)$ \\
\hline Interaction Frequency & $4.26(1.19)$ & $3.18(1.58)$ & $3.66(0.00)$ \\
\hline Trust Expectation & $12.63(5.86)$ & $9.43(5.69)$ & $3.94(0.00)$ \\
\hline Reciprocity Decision & $1.81(0.52)$ & $1.75(0.61)$ & $1.42(0.17)$ \\
\hline
\end{tabular}

Notes:

'Standard deviations are in parentheses.

procedure (Brislin, 1980). In part two, we replicated the hypothetical trust game as carried out in Study 1. Participants were also paid a fixed participation fee of $¥ 10$ RMB.

Manipulation checks. To ensure that participants did consider their relationship with their classmates to be shouren guanxi while those with a non-classmate to be shengren guanxi, we examined participants' responses to the shouren/shengren guanxi and interaction frequency scales. Results show that participants did perceive their relationship with a classmate to be significantly closer than that with a non-classmate (mean $=4.72$ vs. 3.19 , respectively, $F(1,59)=41.23, p=0.00$ ). Similarly, the interaction frequency was also rated as significantly higher (mean $=4.88$ vs. 3.59 , respectively, $\mathrm{F}(1,59)=34.56, \mathrm{p}=0.00)$. These results confirm that our experimental manipulation varied the perceived social distance of the anonymous counterpart.

\section{RESULTS - STUDY 2}

We first verified that the results of Study 2 were consistent with those from Study 1. As Table 3 shows, using a one-sample $t$-test, we observed that as in Study 1, differing social distance levels produced significant within-person differences in both trust decisions and expectations ( $p=0.00$ and 0.00 , respectively) but not in either reciprocity measure. We then conducted mixed ANOVA tests using 
Table 4. Mixed ANOVA testing effects of social distance and order (Study 2)

\begin{tabular}{|c|c|c|c|c|}
\hline Variable and Source & $d f$ & $F$ & p-value & Partial $\eta^{2}$ \\
\hline \multicolumn{5}{|l|}{ Pancl A: Trust Decision } \\
\hline \multicolumn{5}{|l|}{ Model I } \\
\hline Social Distance & 1 & 21.62 & 0.00 & 0.44 \\
\hline Social Distance*Order & 1 & 0.15 & 0.70 & 0.01 \\
\hline Error & 28 & & & \\
\hline \multicolumn{5}{|l|}{ Model 2} \\
\hline Social Distance & 1 & 5.73 & 0.02 & 0.19 \\
\hline Social Distance*Affect-Based Trust & 1 & 18.60 & 0.00 & 0.43 \\
\hline Social Distance*Order & I & 0.39 & 0.54 & 0.02 \\
\hline Error & 27 & & & \\
\hline \multicolumn{5}{|l|}{ Panel B: Trust Expectation } \\
\hline \multicolumn{5}{|l|}{ Model 1} \\
\hline Social Distance & 1 & 15.00 & 0.00 & 0.35 \\
\hline Social Distance*Order & 1 & 0.06 & 0.81 & 0.00 \\
\hline Error & 28 & & & \\
\hline \multicolumn{5}{|l|}{ Model 2} \\
\hline Social Distance & 1 & 2.82 & 0.11 & 0.10 \\
\hline Social Distance*Affect-Based Trust & 1 & 35.47 & 0.00 & 0.57 \\
\hline Social Distance*Order & 1 & 0.99 & 0.33 & 0.04 \\
\hline Error & 27 & & & \\
\hline \multicolumn{5}{|l|}{ Panel C: Reciprocity Decision } \\
\hline Social Distance & 1 & 0.93 & 0.35 & 0.03 \\
\hline Social Distance*Trust Expected & 1 & 0.09 & 0.77 & 0.00 \\
\hline Social Distance*Order & 1 & 0.10 & 0.76 & 0.00 \\
\hline Error & 26 & & & \\
\hline \multicolumn{5}{|l|}{ Panel D: Reciprocity Expectation } \\
\hline Social Distance & 1 & 0.74 & 0.40 & 0.03 \\
\hline Social Distance*Trust Received & 1 & 0.20 & 0.66 & 0.01 \\
\hline Social Distance*Order & 1 & 1.33 & 0.26 & 0.05 \\
\hline Error & 24 & & & \\
\hline
\end{tabular}

the Study 2 data. The results are presented in Table 4. As shown in Model 1 for trust measures, there was a significant main effect of social distance. In particular, participants indicated that they would send significantly more money, our measure of trust, to a classmate than to a non-classmate $[\mathrm{F}(1,28)=21.62, \mathrm{p}=0.00]$, and would expect others to treat them differently in this respect as well $[\mathrm{F}(1,28)=15.00, \mathrm{p}=0.00]$.

In contrast, for reciprocity decisions and expectations, no significant main effect of social distance was found. Moreover, neither order nor difference in trust received interacted significantly with social distance. Thus Study 2 successfully replicated Study 1, providing further support for $\mathrm{H} 1 \mathrm{~b}$ and $\mathrm{H} 3 \mathrm{~b}$.

Next, we observed that affect-based trust was significantly greater for classmates than for non-classmates $(p=0.00)$; while no such effect was found for cognition-based trust. Thus, Hypothesis 4 a was corroborated. We then tested the 
mediation Hypothesis $4 \mathrm{~b}$ following the procedures outlined by Baron and Kenny (1986). First, we established the existence of a main effect of social distance on trust. Second, we observed that affect-based trust was significantly higher for classmates than for non-classmates $(p=0.00)$ and this variation predicted both trust decisions and expectations ( $p=0.00$ for both cases), establishing affect-based trust as a candidate for mediation. Third, when both social distance and affectbased trust were used to predict trust decisions and expectations, the effect of social distance diminished $(p=0.02$ and $p=0.11$ for decisions and expectations, respectively), while the effect of affect-based trust difference retained its level of significance $(p=0.00$ for both hypothetical and expected trust). Moreover, partial $\eta^{2}$ fell from 0.44 to 0.19 for hypothetical trust and from 0.35 to 0.10 for expected trust when affect-based trust was added to the analysis. These results demonstrate that the affect-based trust felt toward a counterpart mediates the effect of social distance on trust decisions and expectations in an economic exchange, corroborating Hypothesis 4b.

\section{DISGUSSION}

Our two studies yielded a very clear pattern of findings. We discuss the key findings below and compare them with the findings of prior research.

First, participants exhibit more trust toward classmates than non-classmates. This shows in behavioural, hypothetical, and expectation measures. This result contrasts with Buchan et al.'s (2006) perplexing result that Chinese participants exhibit more trust toward outgroup members. One key methodological difference was likely responsible for these contrasting results. Unlike the minimal-group paradigm for the manipulation of social distance levels in Buchan et al. (2006), our manipulation varied counterparts in two naturally occurring relationship categories, classmate shouren and non-classmate shengren. Cross-cultural research suggests that the minimal group paradigm provides a less compelling simulation of a relationship in less individualistic cultures where groups are slower to form (Mann, Radford, \& Kanagawa, 1985). More importantly, in the Chinese context the shouren-shengren distinction is salient among natural groups in collective societies, it may be less pronounced for ad hoc groups that are temporarily constructed in a laboratory setting (Triandis, 1995). Thus, as Buchan et al. (2006) conjectured, it might be more difficult to form temporary ad hoc ingroups among Chinese participants due to China's collectivist culture. The fact that our results concerning trust were consistent with Buchan and Croson's (2004) survey data, which also employed naturally occurring relationship categories, lends further support to the notion that this is a critical factor in the examination of the impact of guanxi on trust in China.

Second, in stark contrast to trust, reciprocity was not sensitive to social-distance manipulation. This lack of treatment effect appeared with behavioural, hypotheti- 
cal, and expectation measures. In other words, while non-classmates were actually as reciprocating as classmates, and furthermore expected to be so, they nonetheless received less trust. ${ }^{[4]}$ This result is contrary to the results in Buchan and Croson (2004), who found a significant drop in reciprocity as social distance increased in their hypothetical questionnaire data. We conjecture that this apparent inconsistency is rooted in Buchan and Croson's use of a different reciprocity measure in conjunction with their omission of trust received as a control variable. ${ }^{[5]}$

We hypothesized, and subsequently demonstrated empirically in Study 2, that the distinctive effect of social distance on trust (as opposed to reciprocity) was mediated by higher levels of affect-based trust felt toward the shouren classmates relative to the shengren non-classmates. As discussed earlier, while cognition-based trust refers to trust arising from a rational appraisal of how trustworthy or reciprocating a counterpart will be, affect-based trust has more to do with the emotional and social bonds one has with another person. From this perspective, it is not surprising that trustors sent more money to classmates with whom such bonds are stronger than to non-classmates with whom such bonds are weaker.

Third, while the level of trust was influenced by the closeness of the affective bonds between trustor and trustee, the level of reciprocity was not. When making trust decisions, there is always some vulnerability because of the uncertainty about how the trustee will respond. In such a context, affective bonds can have an important impact that goes beyond purely cognitive instrumental concerns. However, reciprocity decisions are made in response to a known amount of trust that has already been tendered. In the context of such certainty, affective bonds appear to make little difference as social distance had no significant impact on reciprocity ratio levels.

Lastly, our results show that decisions and expectations in behavioural games with real incentives were parallel to those in hypothetical survey scenarios. That both the social-distance effect on trust and the lack of such an effect on reciprocity were robust to different investigative approaches gives added support and confidence to the important relationship between social distance and trust in China.

\section{Contributions to Theory}

Our article makes three important theoretical contributions. First, it enriches the current understanding of guanxi. Tsui and Farh (1997) provided an excellent theoretical discussion of the nuanced nature of the distinction between guanxi and relational demography. Since classmates and non-classmates were anonymous and demographically identical in our study, treatment differences were due only to the differing types of guanxi attached to the classmate versus the non-classmate relationship. While the relational demography theory would predict no difference in trust between anonymous classmates versus anonymous non-classmates, the guanxi theory contends that there would be a difference. In particular, the guanxi theory 
suggests that demographic factors are confounded with shared experiences, producing affective trust, and will be associated with more behavioural trust than would the same demographic factors in the absence of such shared experiences. Our empirical results provide strong support for guanxi theory.

Second, our results support the idea that the magnitude of the affect-based component of trust depends on the type of guanxi between the interacting parties, while cognitive-based trust does not differ significantly between the shouren guanxi and shengren guanxi categories in the presence of a common social identity based on relational demography. Affective bonds neither led trustors to predict a higher level of reciprocity from trustees, nor led trustees to reciprocate more generously in response to trust received. However, trustors were nonetheless willing to send more money to a shouren classmate than to a shengren non-classmate even though trustors' cognition-based predictions of reciprocity were the same for each. This willingness appears to be based on affect rather than cognition. In essence, we brought together constructs from the literatures on trust in managerial relationships to the economic and game-theory literatures on trust and reciprocity issues. This is a noteworthy theoretical contribution.

That said, affect-based trust only partially mediates the relationship between social distance and trust decisions made in hypothetical conditions. One possible explanation for the portion of the social-distance effect that does not work through affect-based trust is that while point predictions of classmate versus non-classmate reciprocity are no different statistically, the unobserved confidence intervals surrounding these predictions might differ. If risk-averse trustors are less certain about how much will be sent back by non-classmate trustees, they might decide to send less even though the expected value of non-classmate reciprocity may not differ from that of classmates. Whether or not this is the case cannot be determined by the point estimates of reciprocity gathered in our study. However, it should be pointed out that the standard deviations of actual reciprocity behaviour reported in Table 1 are no larger for non-classmate reciprocity than for classmate reciprocity. Thus, there appears to be no obvious evidence for a confidence interpretation of the reciprocity patterns. This issue deserves further study.

Third, in contrast to the observed differences in trust, the reciprocity ratio did not change with social distance. In other words, in contrast to trust itself, the response to trust is no different between shouren classmates than between shengren non-classmates with a shared demographic identity. This implies that while guanxi significantly affects trust, it may not affect reciprocity. Our findings suggest that trust-related judgements and decisions are driven by affective concerns, which are greater felt toward shouren than shengren. In contrast, reciprocity-related judgements and decisions do not seem to be driven by such sentiments, as they are not greater for shouren than for shengren. This presents a challenge to accounts of guanxi as a unitary factor affecting all sorts of economic decisions. Different aspects of guanxi, aside from affect-based trust, may be important as drivers of reciprocity decisions. 


\section{Implications for Practice}

Guanxi is still of paramount importance for doing business in the midst of China's transformation from a centrally planned to a socialist market economy. In China, trust embodies social, emotional, and relational elements (Lewicki, McAllister, \& Bies, 1998), and flows more readily when it is rooted in closer guanxi. Our article reinforces the insights of earlier research using different methodologies regarding the importance of building family-like, affect-based guanxi relationships within organizations and between persons doing business with each other in China (e.g., Chua et al., 2009; Ng \& Chua, 2006; Su \& Littlefield, 2001). As also shown in the different context of a social dilemma game by $\mathrm{Ng}$ and Chua (2006), the affect-based component of shouren guanxi is an effective means of building trust-based cooperation. In our studies, the average level of the reciprocity ratio was well above one for classmates and non-classmates alike. Thus, more trust not only created social surplus, i.e., more overall benefit for the trustor-trustee pair, through the tripling mechanism, but was also rewarding on average for trustors individually. It is likely to be so outside the lab as well, especially when supported by affective ties and the principle of escalating reciprocity that is an ingrained part of the guanxi system (c.g., Su \& Littlefield, 2001). Thus, we urge potential investors and foreign managers to actively build guanxi and develop initiatives to build trust and reciprocity relationships. Our findings provide evidence that cultivating personal relationships builds trust.

While some researchers have warned about the negative aspects of the guanxi system in that it can contribute to nepotism and corruption in Chinese society (c.g., Chen, Chen, \& Xin, 2004), our findings suggest that guanxi can have a positive effect as well by engendering a higher level of affect-based trust, which is likely to bring positive outcomes to both interacting parties. Su and Littlefield (2001) provide a very insightful discussion directed at Western managers about how to engage in the building of guanxi, while avoiding the traps posed by unethical practices.

\section{Limitations and Directions for Future Research}

The current research has a number of limitations. First, trust and reciprocity arise in many different contexts in the world outside the lab. We employed a laboratory trust game in our article. The relative simplicity of the game's structure, while allowing us to isolate the concepts of interest, does not permit us to conclude that identical behaviour would be observed in natural business environments with their many subtle complexities. Moreover, an economic exchange with another person is only one form of trust among many. We focused on the trust game because it is one of the simplest and most direct ways of observing and measuring actual trust and reciprocity behaviour, and thus a good place 
to begin examining the effect of guanxi on judgements and behaviour predicted by theory. Although the results of this study corroborate the theoretical predictions regarding guanxi on trust and illustrate a contrasting lack of effect on reciprocity in an economic context, their applicability to the many other forms of trust and reciprocity that exist in the workplace is uncertain. It is important to examine the effects of guanxi on other forms of trust and reciprocity in future research.

Second, this research focuses on just two levels of social distance: classmate shouren and non-classmate shengren with a common demographic identity. It is also important to examine how other relationships between people affect trust and reciprocity. Similarly, we employ a minimal guanxi paradigm, maintaining anonymity between participants to remove perceptions individuals may have of each other and social pressure. In future work, it would be interesting and informative to investigate how removing anonymity either before or after the trust and reciprocity exchanges and/or making individual trust and reciprocity decisions public might interact with social distance to affect behaviour.

Third, following a mono-cultural indigenous approach, we presented an in-depth one-country study, rather than a cross-cultural comparative study. Monocultural indigenous research 'attempts to understand individual psychological functioning in the cultural context in which it developed' (Berry, 1994: 120). Hence it emphasizes 'the roles cultural traditions and social practices play in regulating, expressing, transforming and permuting the human psyche' (Shweder, 1990), as well as the interactions between culture and the human mind (Shweder, 1990). Indigenous inquiries such as ours aim to develop an evolving system of knowledge specific to that culture, which will ultimately produce a higher-order, balanced, global understanding of human cognition and behaviour (Yang, 2000). Indeed, we speculate that the organizational structure that promotes particular closeness among classmates in China is itself a reflection of the central importance of guanxi in Chinese culture. Nonetheless, it would be interesting to examine other societies to see how social distance affects trust in differing cultural contexts. For example, do other countries influenced by Confucian Chinese culture, like Japan and Korea, show similar patterns to those observed in China? What role does quanxi play in overseas Chinese communities? How does social distance affect trust and reciprocity in other non-Western countries? These are all interesting areas for future research.

Notwithstanding the limitations discussed above, the methodology employed in our paper created a controlled laboratory environment, enabling us to abstract from confounding factors to isolate the effects of social distance on trust and reciprocity in a culture of guanxi. It also employed a hypothetical questionnaire to probe beyond behaviour to consider the attitudes and expectations underlying exchanges of trust and reciprocity between shouren and between shengren with a common demographic identity. 


\section{CONCLUSION}

A society cannot advance without trust and reciprocity. Earlier literature has emphasized that trust is highly influenced by cultural heritage and social institutions. In the context of China, the consistent and complementary results obtained using two methodological approaches in this study suggest that guanxi can foster a higher level of affect-based trust. Closer and deeper guanxirelationships engender such trust, while not necessarily affecting reciprocity. This can promote the creation of social surplus. At the levels of reciprocity observed in our study, both trustors and trustees benefit from this surplus even though the level of reciprocity is not itself directly affected by social distance. This suggests a potential to extend the boundaries of trust in China, creating benefits for individuals and organizations both as trustors and trustees. While China has enjoyed tremendous economic and social achievement in the last three decades, further and sustainable long-term progress will depend on cultivating and fostering higher levels of trust and reciprocity within Chinese society and between Chinese and potential collaborators abroad. Meanwhile, understanding guanxi is an important priority for those doing business in China.

\section{NOTES}

We would like to note that the first two authors made an equal contribution to this article. We thank participants at the Economic Science Association meeting and the Trust Manuscript Workshop at the 2007 Academy of Management Conference for their helpful comments and suggestions. Wc also thank the Editor-in-Chief of Management and Organization Review, Anne Tsui, Senior Editor Michacl Morris, and two anonymous reviewers, each of whom offered many interesting and stimulating suggestions that substantially improved the paper. We also thank Rachel Croson and other participants at the Economic Science Association conference for their helpful comments and suggestions. Financial support was provided by the Social Sciences and Humanities Research Council of Canada, grant \#2006-410-138 (Song) and \#2001-410-159 (Cadsby).

[1] Initially, we controlled for possible two-way interactions between gender and social distance as well as gender and order, and a possible three-way interaction between gender, order, and social distance as well. None of the independent variables involving gender was ever significant at conventional levels. Thus, gender was dropped from the analysis.

[2] Earlier work has provided mixed evidence regarding whether the level of trust received can influence the level of the reciprocity ratio in sequential-cxchange games. For example, using the same trust game, Pillutla, Malhotra, and Murnighan (2003) found that reciprocity ratios increased with the level of trust, while others reported that trustees do not respond to an increase in trust experienced by a higher reciprocity ratio (e.g., Bolton, Brandts, \& Ockenfels, 1998; Charness \& Rabin, 2002; Song, 2008, 2009). Given such contrasting empirical results reported in earlier papers, we felt it prudent to control for the level of trust received.

[3] We thank Michael Morris, Senior Editor of Management and Organization Review, for suggesting this study.

[4] A similar apparent disconnection between trustors and trustecs is reported by Cox and Deck (2005) in a different kind of trust game in which first movers choose either to engage or exit while second movers choose cither to cooperate or defect. Decreasing anonymity leads to a higher rate of cooperation by second movers, but has no effect on the rate at which first movers choosc to engage.

[5] Buchan and Croson (2004) defined reciprocity differently from the way it was defined in our paper. In particular, while we defined reciprocity as the ratio of the amount sent back by the trustec to the amount sent by the trustor, Buchan and Croson (2004) defined it as the ratio 
between the amount sent back and the total post-transfer wealth of the trustee. Thus, the denominator of the Buchan and Croson (2004) ratio was the sum of the trustee's initial endowment plus the tripled amount received. Moreover, Buchan and Croson (2004) did not control for the level of trust received at each social distance level. We did have such a control and found that the difference in trust received at each level of social distance had no significant impact on the corresponding difference in reciprocity using our definition; however this implies that it must have an impact on the reciprocity measure employed by Buchan and Croson (2004). Let $\mathrm{E}=$ Endowment of the Trustee, $\mathrm{S}=$ Amount Sent by the Trustor, and $\mathrm{B}=$ Amount Sent Back by the Trustee. Then our ratio $=\mathrm{R} 1=\mathrm{B} / \mathrm{S}$ and the Buchan and Croson ratio $=\mathrm{R} 2=$ $\mathrm{B} /[(\mathrm{E}+3 \cdot \mathrm{S})]$. Suppose $\mathrm{Rl}$ is a constant, i.e., it does not change as $\mathrm{S}$ changes. Then $\frac{\delta R 2}{\delta S}=R 1 \cdot\left[\frac{E}{(E+3 \cdot S)^{2}}\right]>0$. Thus, a constant $\mathrm{R} 1$ implies that $\mathrm{R} 2$ increases with the amount sent. Using our data together with Buchan and Croson's (2004) reciprocity ratio, the difference in trust received does indeed have such an impact. However, as with our ratio, there is no significant relationship between social distance and reciprocity. If the difference in trust received is erroneously omitted from the analysis, an apparent significant relationship between social distance and reciprocity appears in our data. This reflects omitted variable bias. Since classmates tender more trust than non-classmates, this implies a higher Buchan and Croson reciprocity ratio when there is no control for trust received. We conjecture that this may be the case with Buchan and Croson's (2004) data as well. If so, it would explain why their findings regarding social distance and reciprocity differ from ours.

\section{REFERENCES}

Akerlof, G. A. 1997. Social distance and social decisions. Econometrica, 65(5): 1005-1027.

Baron, R. M., \& Kenny, D. A. 1986. The moderator-mediator variable distinction in social psychological research: Conceptual, strategic, and statistical considerations. Journal of Personality and Social Psychology, 51(6): 1173-1 182.

Berg, J., Dickhaut, J., \& McCabe, K. 1995. Trust, reciprocity, and social history. Games and Economic Behavior, 10(1): 122-142.

Berry, J. W. 1994. An ecological perspective on cultural and ethnic psychology. In E. J. Trickett, R. J. Watts \& D. Birman (Eds.), Human diversity: Perspective on people in context: 115-141. San Francisco, CA: Jossey-Bass.

Bian, Y. 1997. Bring strong ties back in: Indirect tics, network bridges, and job searches in China. American Sociological Review, 62(3): 366-385.

Bolton, G. E., Brandts, J., \& Ockenfels, A. 1998. Measuring motivations for the reciprocal responses observed in a simple dilemma game. Experimental Economics, 1(3): 207-219.

Bond, M. H. 1991. Beyond the Chinese face: Insights from psychology. Oxford: Oxford University Press.

Brislin, R. W. 1980. Translation and content analysis of oral and written material. In H. C. Triandis \& J. W. Berry (Eds.), Handbook of cross-cultural psychology: 389-444. Boston, MA: Allyn \& Bacon.

Buchan, N., \& Croson, R. 2004. The boundaries of trust: Own and others' actions in the U.S. and China. Journal of Economic Behavior and Organization, 55(4): 485-504.

Buchan, N., Johnson, E. J., \& Croson, R. 2006. Let's get personal: An international examination of the influence of communication, culture and social distance on other regarding preferences. Journal of Economic Behavior and Organization, 60(3): 373-398.

Burt, R. S. 1992. Structural holes: The social structure of competition. Cambridge, MA: Harvard University Press.

Butterfield, F. 1982. China: Alive in the bitter sea. New York: Times Books.

Camerer, C. F. 2003. Behavioral game theory: Experiments in strategic interaction. Princeton: Princeton University Press.

Chai, S. K., \& Rhee, M. 2010. Confucian capitalism and the paradox of closure and structural holes in East Asian firms. Management and Organization Revieze, 6(1): 5-29.

Charness, G., \& Rabin, M. 2002. Understanding social preferences with simple tests. Quarterly Journal of Economics, 117(3): 817-869. 
Chen, X. P., \& Chen, C. C. 2004. On the intricacies of the Chinese guanxi: A process model of guamxi development. Asia Pacific Joumal of Management, 21(3): 305-324.

Chen, C. C., Chen, Y. R., \& Xin, K. 2004. Guanxi practices and trust in management: A procedural justice pcrspective. Organization Science, 15(2): 200-209.

Chiao, C. 1982. Guanxi: A preliminary conceptualization. In K. S. Yang \& C. I. Wen (Eds.), The sinicization of social and behavioral science research in China: 345-360. Taipei: R.O.C.: Academia Sinica.

Child, J., \& Mollering, G. 2003. Contextual conficlence and active trust development in the Chinese business environment. Organization Science, 14(1): 69-80.

Chua, R. Y.J., Morris, M. W., \& Ingram, P. 2009. Guanxi vs. networking: Distinctive configurations of affect- and cognition-based trust in the networks of Chinese vs. American managers.Journal of International Business Studies, 40(3): 490-508.

Cox, J. C., \& Deck, C. A. 2005. On the nature of reciprocal motives. Economic Inquiry, 43(3): $623-635$.

Farh, J. L., Tsui, A. S., Xin, K., \& Cheng, B. 1998. The influence of relational demography and guanxi: The Chincse case. Organizational Science, 9(4): 471-488.

Fukuyama, F. 1995. Trust: Social virtues and the creation of prosperity. London: Hamish Hamilton.

Garbarino, E., \& Slonim, R. 2009. The robustness of trust and reciprocity across a heterogencous U.S. population. Journal of Economic Behavior \& Organization, 69(3): 226-240.

Granovetter, M. 1985. Economic action and social structure: The problem of embeddedness. American Journal of Sociology, 91(3): 481-510.

Gunnthorsdottir, A., McCabe, K., \& Smith, V. 2002. Using the Machiavellianism instrument to predict trustworthiness in a bargaining game. Journal of Economic Psychology, 23(I): $49-66$.

Guo, C., \& Giacobbe-Miller, J. 2010. Guanxi dynamics and entrepreneurial firm creation and dcvelopment in China. Management and Organization Revieze, 6(2): 267-291.

Hofstcde, G. 1991. Cultures and organizations: Software of the mind. New York: McGrawHill.

Hogarth, R. M., \& Reder, M. W. 1987. Rational choice: The contrast between economics and psychology. Chicago, IL: University of Chicago Press.

Hwang, K. K. 1987. Face and favor: The Chinese power game. American Journal of Sociology, 92(4): 944-974.

Jacobs, J. B. 1979. A preliminary model of particularistic ties in Chinese political alliances: Kan-ch'ing and Kuan-hsi in a rural Taiwanese township. The China Quarterly, 78: 237273.

King, A. Y. 1991. Kuan-hsi and network building: A sociological interpretation. Daedalus, 120(2): 63-84.

Levin, D. Z., \& Cross, R. 2004. The strength of weak tics you can trust: The mediating role of trust in effective knowledge transfer. Management Science, 50(11): 1477-1490.

Lewicki, R. J., \& Bunker, B. B. 1995. Trust in relationships: A model of trust development and decline. In B. B. Bunker \& J. Rubin (Eds.), Conflict, cooperation and justice: Essays inspired by the work of Morton Deutsch, The Jossey-Bass management series and the Jossey-Bassy conflict resolution series: 122-173. San Francisco, CA: Jossey-Bass.

Lcwicki, R. J., McAllister, D.J., \& Bies, R. J. 1998. Trust and distrust: New relationships and realities. Academy of Management Reviere, 23(3): 438-458.

Li, S. X., Yao, X., Sue-Chan, C., \& Xi, Y. 2011 . Where do social ties come from: Institutional framework and governmental tie distribution among Chinese managers. Management and Organization Review, 7(1): 97-124.

Loewenstein, G. 1996. Out of control: Visceral influences on behavior. Organizational Behavior and Human Decision Processes, 65(3): 272-292.

Luo, Y. D. 2000. Guanxi and business. Singapore: World Scientific Publishing.

Mann, L., Radford, M., \& Kanagawa, C. 1985. Cross-cultural difference in children's use of decision rules: A comparison between Japan and Australia. Journal of Personality and Social Psychology, 49(6): 1557-1564.

McAllister, D. J. 1995. Affect- and cognition-based trust as foundations for interpersonal cooperation in organizations. Academy of Management Journal, 38(1): 24-59. 
Mayer, R. C., Davis, J. H., \& Schoorman, F. D. 1995. An integrative model of organizational trust. Academy of Management Reviere, 20(3): 709-734.

Murnighan, J. K. 1994. Game theory and organizational behavior. In B. M. Staw \& L. L. Gummings (Eds.), Research in organizational behavior: 83-123. Greenwich, CT: JAI Press.

$\mathrm{Ng}$, K. Y., \& Chua, R. Y. J. 2006. Do I contribute more when I trust more? Differential effects of cognition- and affect-based trust. Management and Organization Revieze, 2(1): 43-66.

Pillutla, M. M., Malhotra, D., \& Murnighan, J. K. 2003. Attributions of trust and the calculus of reciprocity. Journal of Experimental Social Psychology, 39(5): 448-455.

Pruitt, D. G., \& Kimmel, M. J. 1977. Twenty years of experimental gaming: Critique, synthesis, and suggestions for the future. In M. R. Rosenzweig \& L. W. Porter (Eds.), Annual review of psychology: 363-392. Palo Alto, CA: Annual Reviews.

Rabin, M. 2002. A perspective on psychology and economics. European Economic Revieze, 46(4-5): 657-685.

Robinson, S. L. 1996. Trust and breach of the psychological contract. Administrative Science Quarterly, 41(4): 574-599.

Rotter, J. B. 1967. A new scale for the measurement of interpersonal trust. Joumal of Personality, 35(4): $651-665$.

Rousseau, D. M., Sitkin, S. B., Burt, R. S., \& Gamerer, C. 1998. Not so different after all: A cross-discipline view of trust. Academy of Management Revieze, 23(3): 393-404.

Shweder, R. A. 1990. Cultural psychology: What is it? J. W. Stigler, R. A. Shweder \& G. Herdt (Eds.), Cultural psychology: Essays on comparative human development: 1-46. Cambridge: Cambridge University Press.

Smith, V. L. 1976. Experimental economics: Induced value theory, American Economic Revieze, 66(2): 274-279.

Song, F. 2008. Trust and reciprocity: The differing norms of individuals and group representatives. Games and Economic Behavior, 62(2): 675-696.

Song, F. 2009. The effect of the consensus-making process on intergroup trust and reciprocity in strategic interactions. Organizational Behavior and Human Decision Processes, 108(1): 164-173.

Su, C., \& Littlefield, J. E. 2001. Entering guanxi: A business ethical dilemma in mainland China? Journal of Business Ethics, 33(3): 199-210.

Triandis, H. C. 1995. Individualism and collectivism. Boulder, CO: Westview Press.

Tsui, A. S., \& Farh, J. L. 1997. Where Guanxi matters: Relational demography and guanxi in the Chinese context. Work and Occupations, 24(1): 56-79.

Van Boven, L., Dunning, D., \& Loewenstein, G. 2000. Egocentric empathy gaps between owners and buycrs: Misperceptions of the endowment effect. Joumal of Personality and Social Psychology, 79(1): 66-76.

Wasti, S. A., Tan, H. H., \& Erdil, S. E. 2011. Antecedents of trust across foci: A comparative study of Turkey and China. Management and Organization Revieze, 7(2): 279-302.

Webber, S. S., Chen, G., Marsh, S., \& Payne, S. 1999. Performance appraisal research contributes to team mental model measurement. In S. J. Zaccaro (Chair) \& D. A. Hofmann (Discussant), Breaking barriers in team research: Neze methodologies and applications for team research and practice. Symposium conducted at the 1998 annual meeting of the Academy of Management, San Diego, CA.

Wilson, R. K., \& Eckel, C. C. 2011 . Trust and social exchange. In J. N. Druckman, D. P. Green, J. H. Kuklinski \& A. Lupia (Eds.), Handbook of experimental political science. Boston, MA: Cambridge University Press.

Xin, K. R., \& Pearce, J. L. 1996. Guanxi: Connections as substitutes for formal institutional support. Academy of Management Journal, 39(6): 1641-1658.

Yamagishi, T. 1998. The structure of trust: The evolutionary games of mind and society. Tokyo: Tokyo University Press.

Yang, K. S. 2000. Monocultural and cross-cultural indigenous approaches: The royal road to the development of a balanced global psychology. Asian Journal of Social Psychology, 3(3): 241-263.

Yang, M. M. 1994. Gifts, favors and banquets: The art of social relationships in China. Ithaca, NY: Cornell University Press. 
Fei Song (fsong@ryerson.ca) is an Associate Professor at the Ted Rogers School of Management, Ryerson University, Canada. She received her Ph.D. in Organizational Behaviour/Industrial Relations from the Schulich School of Business, York University. Her research interests include behavioural decision making, cross-cultural issues, and strategic compensation. She has published in the Academy of Management Journal, Organizational Behavior and Human Decision Processes, Games and Economic Behavior, International Joumal of Conflict Management, Experimental Economics, and Joumal of Economic Psychology.

C. Bram Cadsby (bcadsby@uoguelph.ca) is Professor of Economics at University of Guelph, Canada. His Ph.D. in Economics is from MIT. With research expertise in experimental methodology, his current interests include the effect of compensation schemes on workplace behaviour and the role of promises and trust in economic decisions. His research has appeared in such journals as Management Science, Academy of Management Journal, Reviere of Financial Studies, Games and Economic Behavior, and Foumal of Public Economics.

Yunyun Bi (yunyun_bi@hotmail.com) is a portfolio manager at Taiping Asset Management, one of the biggest insurance asset management companics in China. She received her Master's degree in Economics from the University of Guelph, Canada.

Manuscript received: August 17, 2009

Final version accepted: September 23, 2011

Accepted by: Michael W. Morris 